\title{
Analyst
}

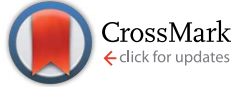

Cite this: Analyst, 2014, 139, 4350

Received 4th November 2013 Accepted 16th June 2014

DOI: $10.1039 / c 3 a n 02067 c$

www.rsc.org/analyst

\section{Determination of psychostimulants and their metabolites by electrochemistry linked on-line to flowing atmospheric pressure afterglow mass spectrometry $\dagger$}

\author{
Marek Smoluch, ${ }^{\text {*a }}$ Przemyslaw Mielczarek, ${ }^{a}$ Edward Reszke, ${ }^{b}$ Gary M. Hieftje ${ }^{c}$ \\ and Jerzy Silberring ${ }^{\text {ad }}$
}

The flowing atmospheric pressure afterglow (FAPA) ion source operates in the ambient atmosphere and has been proven to be a promising tool for direct and rapid determination of numerous compounds. Here we linked a FAPA-MS system to an electrochemical flow cell for the identification of drug metabolites generated electrochemically in order to study simulated metabolic pathways. Psychostimulants and their metabolites produced by electrochemistry (EC) were detected on-line by FAPA-MS. The FAPA source has never been used before for an on-line connection with liquid flow, neither for identification of products generated in an electrochemical flow cell. The system was optimized to achieve the highest ionization efficiency by adjusting several parameters, including distances and angles between the ion source and the outlet of the EC system, the high voltage for plasma generation, flow-rates, and EC parameters. Simulated metabolites from tested compounds [methamphetamine (MAF), para-methoxy- $N$ methylamphetamine (PMMA), dextromethorphan (DXM), and benzydamine (BAM)] were formed in the EC cell at various $\mathrm{pH}$ levels. In all cases the main products were oxidized substrates and compounds after $\mathrm{N}$-demethylation. Generation of such products and their thorough on-line identification confirm that the cytochrome P450 - driven metabolism of pharmaceuticals can be efficiently simulated in an electrochemical cell; this approach may serve as a step towards predictive pharmacology using a fast and robust design.

\section{Introduction}

The flowing atmospheric pressure afterglow (FAPA) ${ }^{\mathbf{1 - 4}}$ and direct analysis in real time $(\mathrm{DART})^{5}$ are electrical-discharge-based devices that operate under ambient conditions and serve as efficient and robust ion sources for mass spectrometers. The distinct feature of these sources is that they do not utilize liquid flow to generate ions. Instead, modest-power plasma forms reagent ions from atmospheric gases, which in turn ionize target analyte species. ${ }^{6,7}$ Further, the hot gases issuing from the source can volatilize many analytes from solid, liquid, or aerosol surfaces directly, thereby eliminating sample preparation. The mechanism of ionization with the FAPA differs from that with

${ }^{a}$ Department of Biochemistry and Neurobiology, Faculty of Materials Science and Ceramics, AGH-University of Science and Technology, Mickiewicza 30, 30-059 Krakow, Poland

${ }^{b}$ ERTEC-Poland, Rogowska 146/5, 54-440 Wroctaw, Poland

${ }^{c}$ Department of Chemistry, Indiana University, Bloomington, Indiana 47405, USA

${ }^{d}$ Centre of Polymer and Carbon Materials, Polish Academy of Sciences, CurieSklodowskiej St. 34, 41-819 Zabrze, Poland

$\dagger$ Electronic supplementary information (ESI) available. See DOI: $10.1039 / \mathrm{c} 3$ an02067c an ESI source. In ESI, ionization proceeds via ion formation from charged droplets while the helium-operated FAPA possesses highly energetic species with an ionization potential as high as $24 \mathrm{eV}$, which interact with the sample material directly or by means of reagent ions generated from atmospheric constituents. Due to the absence of a mobile phase, as in the case of e.g. electrospray ionization (ESI), plasma-based ion sources have not been commonly linked to condensedphase separation systems, such as liquid chromatography. We found only a single report describing the connection of capillary electrophoresis to FAPA. ${ }^{8}$ In contrast, plasma sources have been shown to be an excellent alternative to Electron Ionization (EI) for detection of molecules separated by gas chromatography. ${ }^{9}$ Accordingly, the aim of the present work was to connect liquidbased separation with FAPA to fully utilize the power of an electrochemical cell for simulation of metabolic pathways for thorough and quantitative identification of products generated from several frequently used psychoactive substances.

Studies on drug metabolism are usually performed in vivo, involving live animals or humans, ${ }^{\mathbf{1 0}}$ or in vitro using animals or human liver microsomes. ${ }^{11}$ These latter techniques, although accurately mimicking metabolic reactions that occur in the 
human body, are expensive, time-consuming, laborious, and always raise some ethical issues. Moreover, these methods require extensive purification and separation prior to identification and quantification of all metabolites. Therefore, analytical techniques not involving live organisms attract strong attention. For instance, metabolic pathways can be simulated also by photodegradation by means of a $\mathrm{TiO}_{2} / \mathrm{UV}$ system in aqueous solution. It was shown that $\mathrm{TiO}_{2} / \mathrm{UV}$ oxidation provides complementary results to standard in vivo approaches of metabolism research. ${ }^{12,13}$

It is known that the cytochrome $\mathrm{P} 450$ metabolism of pharmaceuticals present in liver hepatic cells can be efficiently simulated by electrochemical oxidation. ${ }^{\mathbf{1 4 - 1 6}}$ The greatest advantage of such experiments over other in vitro methods to study metabolism is the possibility of combining the electrochemistry system on-line with mass spectrometry detection, which is superior for metabolite detection and identification. The present paper represents the first link of such an electrochemical system with mass spectrometry equipped with a FAPA source, which was applied for determination of the drugs of abuse. Earlier, electrospray (ESI) ionization was frequently the method of choice ${ }^{17}$ however, FAPA can provide important complementary results, in particular for small, hydrophobic compounds that poorly ionize in ESI. ${ }^{1}$ Developments of electrochemistry in combination with mass spectrometry have recently been reviewed ${ }^{18}$ and demonstrate it to be a reliable technique for simulation of metabolites. ${ }^{\mathbf{1 4 - 1 7}}$

The on-line combination of electrochemistry (EC) with mass spectrometry using the flowing atmospheric pressure afterglow (FAPA) ion source provides a novel tool to study psychostimulants and their metabolites in a single-step experiment. This kind of study is important for several reasons. Metabolic processes are still not completely understood; not only these experiments can broaden our knowledge about metabolism, but also provide vital information on general mechanisms of addiction and the influence of drugs on the human body. ${ }^{\mathbf{1 9 , 2 0}}$ Moreover, there is a constant increase in the number of new psychostimulants appearing on the market, with unknown side effects. Identification of metabolites is vital from the point of view of medical care and emergency treatment. Additionally, the increasing number of new substances, particularly legal highs and designer drugs, easily available or home-made produced, become a danger and demands new diagnostic techniques and ways of treatment, especially for adolescents. In this paper we demonstrate the potential applicability of the combination of EC and FAPA where, for the first time, a liquid system is directly introduced into this ion source. This combination extends the capabilities of FAPA in predictive toxicology and forensic sciences. $^{21,22}$

\section{Experimental section}

\section{Reagents and chemicals}

Dextromethorphan (DXM) and benzydamine (BAM) were obtained from Acodin ${ }^{\circledR}$ and Tantum Rosa ${ }^{\circledR}$, respectively. Active compounds were collected by dissolving the tablets in methanol, centrifuging the resultant (2 minutes, $6000 \mathrm{rpm}$, Hettich
Zentrifugen Mikro 22R, Germany), and using the supernatant for further analyses. Methamphetamine (MAF) and paramethoxy- $N$-methylamphetamine (PMMA) were obtained by courtesy of Prof. Andrzej Parczewski (Department of Analytical Chemistry, Jagiellonian University, Krakow, Poland) under permission no.: GIF-N-P/4721/004/2004.

Ammonium formate was obtained from Fluka, ammonium hydroxide, ammonium bicarbonate, acetonitrile and methanol (both reagent grade) were obtained from J. T. Baker. Water was purified by a Simplicity UV Water Purification System (conductivity $18.2 \mathrm{M} \Omega \mathrm{cm}$, Millipore).

Stock solutions of BAM, MAF, PMMA and DXM were prepared in methanol at a concentration of $1 \mathrm{mg} \mathrm{mL}{ }^{-1}$ and used to prepare fresh samples shortly before each experiment, as described below. The procedures were performed under written consent from country authorities in a restricted area of the laboratory.

\section{Instrumentation}

Mass spectrometry. A Bruker Esquire 3000 quadrupole ion trap mass spectrometer (Bruker Daltonics, Bremen, Germany) was used for all measurements. The ionization of all compounds was achieved with a NOVA011 (ERTEC, Wroclaw, Poland) helium-based flowing atmospheric pressure afterglow (FAPA) source. The construction and design of the source have been described elsewhere. ${ }^{23,24}$ The FAPA source was mounted horizontally approx. $1.5 \mathrm{~cm}$ in front of the MS inlet (Fig. 1). The discharge power was adjustable within the range of 3-30 $\mathrm{W}$, and for all experiments the maximal power of $30 \mathrm{~W}$ was used, as it provided the highest signal-to-noise ratio. The open-circuit voltage $(20 \mathrm{kV})$ ensured self-ignition of the helium discharge. Helium (99.999 purity grade) was continuously delivered at a flow rate of $1.0 \mathrm{~L} \mathrm{~min}^{-1}$. The typical ESI-MS source settings were found to be optimal also for the FAPA source, with the exception of the mass spectrometer entrance glass capillary voltage, where a lower potential $(2 \mathrm{kV})$ compared to the standard ESI setting $(4.5 \mathrm{kV})$ was used. The temperature of the glass capillary was set to $280{ }^{\circ} \mathrm{C}$, the drying gas flow was maintained at $5 \mathrm{~L} \mathrm{~min}^{-1}$ and

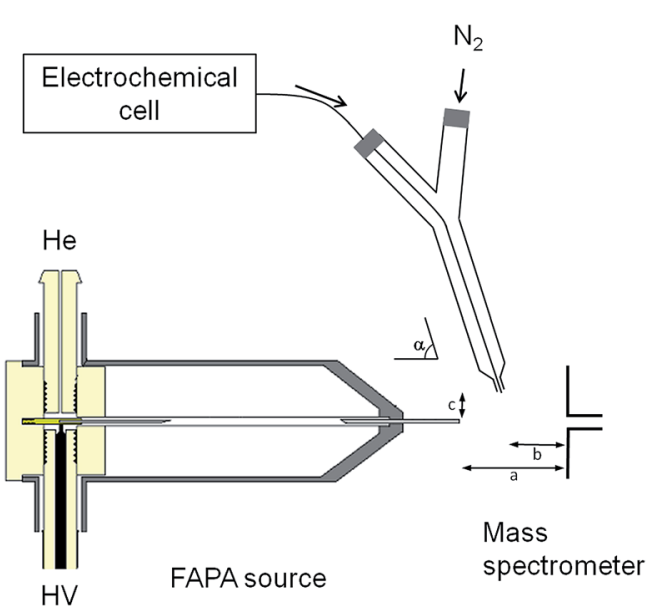

Fig. 1 Schematic diagram of EC-FAPA-MS coupling. See the text for detailed description. 
the nebulizer gas $\left(\mathrm{N}_{2}\right)$ pressure was 15 psi. The scan range was set from 50 to $500 \mathrm{~m} / \mathrm{z}$. For MS/MS experiments the isolation width was set to $1 \mathrm{~m} / \mathrm{z}$ and the fragmentation amplitude was in the range of 0.5 to 1 unit. The geometry of the coupling between the EC and FAPA source is described in the Results section. For EC-ESI-MS experiments the same as above mass spectrometry parameters were applied, except the glass capillary voltage where a potential of $4.5 \mathrm{kV}$ was used.

Electrochemistry (EC). The EC system used for the experiments was the ROXYTM potentiostat with a Dual-Piston Syringe Pump (Antec, Zoeterwoude, The Netherlands). This is one of the first applications of the EC system for generation of metabolites produced from psychoactive compounds. ${ }^{25}$ As a result, several working electrodes were evaluated for this purpose. The first was a glassy carbon electrode (GC, from Antec, Zoeterwoude, The Netherlands) and the alternative was a boron-doped diamond electrode (MD - Magic Diamond ${ }^{\mathrm{TM}}$ electrode from Antec, Zoeterwoude, The Netherlands) consisting of an ultra-thin crystalline diamond layer deposited on top of a silicon substrate. The boron-doped diamond (BDD) electrode exhibits excellent inertness, and can be used with a large variety of mobile phases and chemicals. ${ }^{26}$ Additionally, due to their high overpotential for oxygen, GC and BDD can be used over an extended potential range when oxidation reactions are performed. During the present experiments, when high potentials were applied, we did not observe any instability in the mass spectrometry signal, which indicated that gases were generated in the electrochemical cell and that hydrolysis of water occurred. ${ }^{17,27}$

Stock solutions ( $1 \mathrm{mg} \mathrm{mL}^{-1}$ ) of BAM, MAF, PMMA and DXM were diluted with a buffer solution (composition described below) to a final concentration of $0.1 \mathrm{mg} \mathrm{mL}^{-1}$ for the on-line EC-FAPA-MS experiments with a glassy carbon working electrode and $0.01 \mathrm{mg} \mathrm{mL}^{-1}$ for experiments with the boron-doped diamond working electrode. Both electrodes were tested as they generate distinct products. Three electrolytes of different $\mathrm{pH}$ values were used for electrochemical oxidation: (1) $50 \mathrm{mM}$ ammonium bicarbonate, 50\% acetonitrile, pH 9.0; (2) $20 \mathrm{mM}$ ammonium formate, $50 \%$ acetonitrile, $\mathrm{pH} 7.4$; (3) $0.1 \%$ formic acid, $50 \%$ acetonitrile, $\mathrm{pH} 2.0$.

Solutions of all analyzed compounds in all solvents were electrochemically oxidized at a flow rate of $10 \mu \mathrm{L} \mathrm{min} \min ^{-1}$ in the ROXYTM $^{\mathrm{TM}}$ potentiostat equipped with a thin-layer ReactorCell ${ }^{\mathrm{TM}}$ (Antec, Zoeterwoude, The Netherlands). The volume of the electrochemical cell was $0.75 \mu \mathrm{L}$. A three-electrode configuration was used during the experiment, i.e. the working electrode, the HyREFTM (Antec, Zoeterwoude, The Netherlands) palladium-hydrogen $\left(\mathrm{Pd} / \mathrm{H}_{2}\right)$ reference electrode, and the auxiliary graphite electrode. The glassy carbon working electrode was finished with a polishing disc and diamond slurry provided by the manufacturer (Antec, Zoeterwoude, The Netherlands) prior to each experiment. The boron-doped diamond working electrode (BDD) was primed electrochemically. For this step, a syringe was filled with the mobile phase containing the supporting electrolyte (the same mobile phase used for oxidation of the drugs) and operated at a flow rate of $20 \mu \mathrm{L} \mathrm{min}{ }^{-1}$. The pulsed mode was used for 5 minutes with the following settings:
$E_{1}=+2 \mathrm{~V}, E_{2}=-2 \mathrm{~V}, t_{1}=1000 \mathrm{~ms}$, and $t_{2}=1000 \mathrm{~ms}$. Then the electrode was activated by an oxidative scan from 0 to $1.5 \mathrm{~V}$, at a scan rate of $50 \mathrm{mV} \mathrm{s}^{-1}$, for three cycles. After the activation procedure the cell was turned off and the flow was left on for additional few minutes.

On-line EC/MS experiments were performed by linearly ramping the cell potential from 0 to 2 or $3 \mathrm{~V}$ (depending on the working electrode used in the experiment) at $20 \mathrm{mV} \mathrm{s}^{-1}$ and analyzed using Dialogue software (Antec, Zoeterwoude, The Netherlands). The oxidation products were directly transferred via PEEK tubing (I.D. $0.13 \mathrm{~mm}$; O.D. 1/16 ${ }^{\prime \prime}$ ) and a fused silica capillary (I.D. $0.1 \mathrm{~mm}$; O.D. $0.36 \mathrm{~mm}$ ) to the flowing atmospheric pressure afterglow (FAPA) source and further to the mass spectrometer operated in the positive-ion mode. All connections were made with zero dead volume unions (not grounded). The commercial ESI source had been removed from the mass spectrometer and the high-voltage safety switch blocked with a small magnet. The delay time between product formation within the electrochemical cell and detection in the mass spectrometer was taken into account when the detected product was correlated with the working-electrode potential. The scheme describing all connections is shown in Fig. 1.

\section{Calibration curve preparation for EC-FAPA-MS linking}

For quantitative studies, i.e., estimation of sensitivity and preparation of the standard curve, the EC system was replaced by using an autosampler and a loading pump. For sample injections a Famos autosampler (Thermo Scientific, USA, former LC Packings/Dionex, Amsterdam, The Netherlands) was used. The injection volume was set to $1 \mu \mathrm{L}$ (full loop injection).

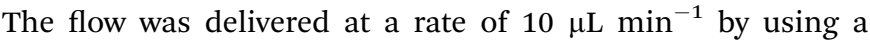
Prominence LC-20AD liquid chromatography pump (Shimadzu, Kyoto, Japan). The mobile phase consisted of $50 \%$ acetonitrile in water. For this study, methamphetamine was selected at the following concentrations: $0.001 ; 0.002 ; 0.005 ; 0.01 ; 0.02$; and $0.05 \mathrm{mg} \mathrm{mL} \mathrm{m}^{-1}$, dissolved in $50 \%$ acetonitrile.

\section{Results and discussion}

\section{Coupling EC to FAPA-MS}

The EC/MS coupling interface was fabricated from plastic parts to avoid current flow between the EC outlet and MS inlet (see Fig. 1); such a current flow would probably lead to electrospray ionization. Even with the use of plastic parts, special precautions must be taken to avoid electrospraying or mixed electrospray/plasma ionization. The reason is that the electrochemical cell operates at a low potential, which can result in a considerable potential difference between the mass spectrometer inlet and the EC outlet (via the mobile phase). To avoid this effect, a safe distance must be maintained between the mass spectrometer inlet and EC outlet. That distance is set experimentally and should not be smaller than $5 \mathrm{~mm}$. Under these conditions the impact of electrospray ionization can be neglected. The geometry of the coupling was optimized for the highest $S / N$ ratio. The FAPA source was placed horizontally $15-20 \mathrm{~mm}$ from the mass spectrometer inlet (distance " $a$ " in Fig. 1). The end of 
the EC capillary outlet (fused silica sticking out from a polyethylene T-piece union) was mounted 2-3 $\mathrm{mm}$ above the mass spectrometer inlet (marked as " $c$ " in Fig. 1) and approximately in the middle of the distance between the mass spectrometer and FAPA source (8-10 $\mathrm{mm}$ from the mass spectrometer inlet " $b$ " in Fig. 1). A nitrogen flow was used to maintain nebulization. The optimal gas pressure of $c a .15$ psi provides the best signal stability and intensity. The angle between the FAPA source and the EC capillary ( $\alpha$ in Fig. 1 ) has a strong impact on spectral quality, and was tested over the range from 30 to 120 degrees. The highest $S / N$ was achieved when $\alpha$ was set to $c a .60^{\circ}$.

\section{Coupling EC to ESI-MS}

The outlet of the EC system was directly coupled to the standard ESI source by means of $50 \mathrm{~cm}$ long PEEK tubing (130 $\mu \mathrm{m}$ I.D.).

\section{Signal stability, linearity and limit of detection}

In order to verify the stability of the EC-FAPA coupling, the mass spectrometer baseline was measured for $60 \mathrm{~min}$. An extracted ion chromatogram (EIC) of $\mathrm{m} / z=195.1$, corresponding to protonated caffeine $\left(50 \mu \mathrm{g} \mathrm{mL}^{-1}\right)$, was used for calculations. The result reveals that signal deviation of the extracted ion chromatogram (not smoothed) do not exceed 10\% (RSD) over the time tested. Caffeine was applied in this particular case to avoid unnecessary consumption of methamphetamine, as this experiment has no impact on further optimization of the system.

A calibration curve was prepared by injecting methamphetamine with the aid of an autosampler at the following concentrations: $1 ; 2 ; 5 ; 10 ; 20 ; 50 \mu \mathrm{g} \mathrm{mL}{ }^{-1}$. At each concentration $1 \mu \mathrm{L}$ injection was repeated three times and the calculated peak areas were averaged with an RSD not exceeding $20 \%$ for each concentration. Good linearity within the experimental range was observed with the following linear equation: $y=447000 x-$ $10^{6}$ and $R^{2}=0.995$. Based on the above experiments, the LOD for methamphetamine was estimated at $2.5 \mu \mathrm{g} \mathrm{mL}^{-1}$. The calculations were made using the $S / N$ ratio given by the Data Analysis software for each calibration point and comparison to a blank region of the mass spectrum.
There are several papers showing extremely sensitive analyses using FAPA for some groups of compounds ${ }^{28}$ but, in general, the sensitivity of the FAPA source (as well as other sources) is strongly compound-dependent. The psychostimulants studied in the present paper seem not to be the ideal candidates for achieving extremely good LODs, but the goal of our work was to show applicability of the entire EC/FAPA system as a part of predictive toxicology, where higher quantities of illegal synthetic substances are available.

\section{Identification of metabolites}

In order to obtain products generated electrochemically from psychostimulants, the substrates were introduced into the electrochemical cell at a flow rate of $10 \mu \mathrm{L} \mathrm{min}{ }^{-1}$. Depending on the $\mathrm{pH}$ of the solvent, various metabolites were observed ( $c f$. Table 1). In all cases, mainly two types of products are observed: one corresponding to an increase in mass by $14 \mathrm{Da}$, and the second showing mass reduction by $14 \mathrm{Da}$. An increase in mass can be explained by a two-step process, consisting of oxidation, followed by dehydrogenation, although in the case of DXM only a dehydrogenated compound $(-2 \mathrm{~m} / \mathrm{z})$ is observed. The second case involves a reduction in mass by 14 Da and can be explained by a loss of $\mathrm{CH}_{2}$ as a result of $\mathrm{N}$-demethylation. In the case of DXM, a decrease in $m / z$ of 16 is seen, corresponding to dehydrogenation and demethylation. The proposed structures of the identified oxidation products are shown in Fig. 2. The structures were deduced on the basis of the EC-FAPA-MS and EC-ESI-MS experiments (hydroxylation of MAF, data not shown) and data available in the literature for PMMA ${ }^{29,30}$ and MAF. ${ }^{31}$ The structures for BAM and DXM are not shown, although the literature indicates that the main metabolites for these compounds are $\mathrm{N}$-demethylated and $\mathrm{N}$ (ring)-oxygenated products, ${ }^{32,33}$ and $\mathrm{O}$ - and $\mathrm{N}$-demethylated products, ${ }^{34}$ respectively. The formation of oxidation products can be clearly detected by monitoring the extracted ion chromatograms (EIC) for selected ions of interest (Fig. 3).

A voltage increase in the electrochemical cell leads to oxidation of the substrate and results in a reduction in signal of the ion corresponding to the substrate. Simultaneously, oxidation products are formed and observed as an appearance of the

Table 1 Summary of all products generated by the electrochemical cell and detected by FAPA-MS. $\mathrm{m} / \mathrm{z}$ values have been rounded to the nearest integer

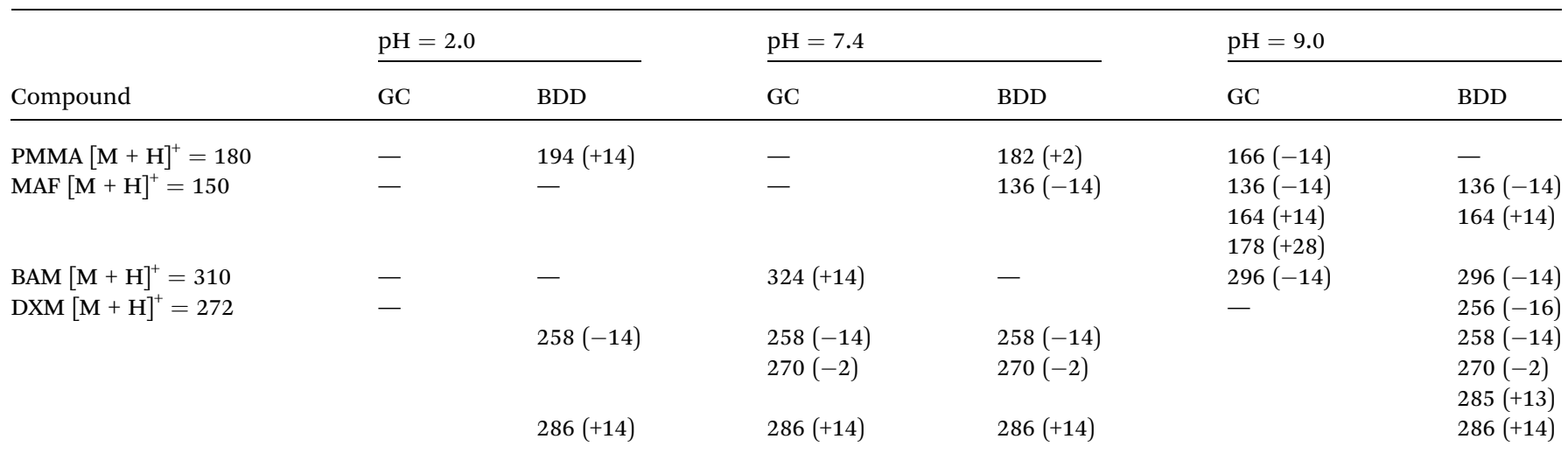


a)

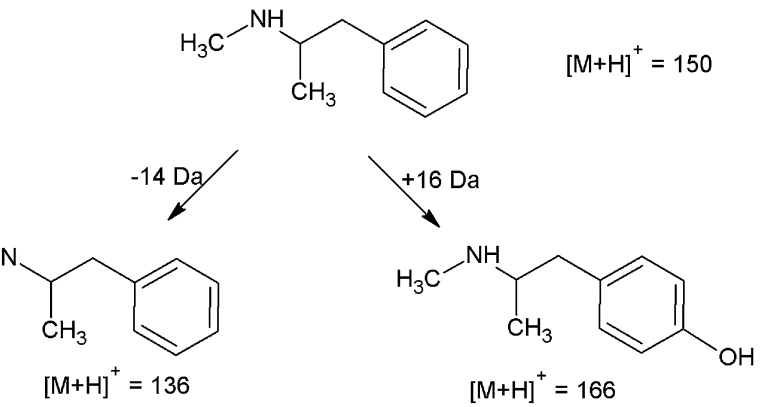

b)

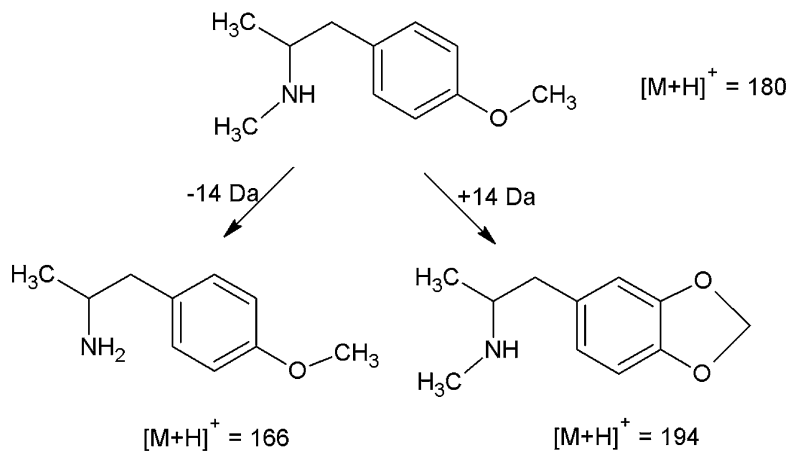

Fig. 2 Structures of the proposed main oxidation products for MAF (a) and PMMA (b). See the text for more details.

target metabolite (see Fig. 3). The maximal conversion of the substrate into the product is observed for voltage range between 1-2.5 $\mathrm{V}$ for the glassy carbon and boron-doped diamond electrodes, although these values may vary, depending on the reaction. The example of EIC for DXM is shown in Fig. 3. Fig. 4 presents the mass spectrum for this compound,

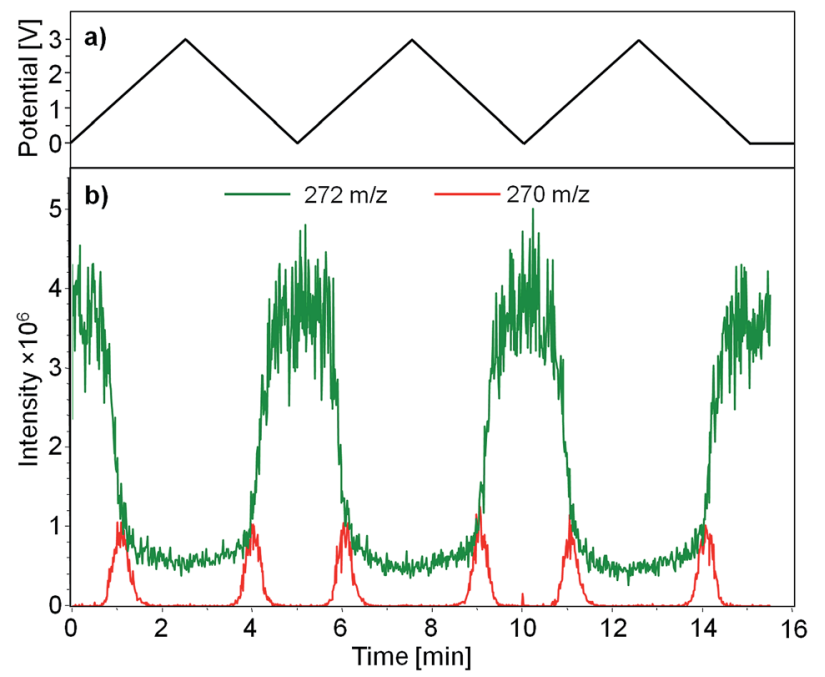

Fig. 3 Example of extracted ion chromatograms for ions at $\mathrm{m} / \mathrm{z} 270$ and 272, resulting from the electrochemical oxidation of dextromethorphan. The signal is dependent on the voltage used in the EC cell (three cell cycles are shown - see graph (a); the maximum conversion rate was achieved for EC voltages in the range of $1-3 \mathrm{~V}$ and the maximum product generation $(\mathrm{m} / \mathrm{z} 270)$ was for ca. $1 \mathrm{~V}$ - see graph (b)). and the MS/MS spectrum of the protonated DXM molecule, together with its oxidation product formed during the electrochemical process.

The concern may arise whether the introduction of the liquid system into the FAPA source may affect the chemistry involved in the mechanism of ionization. This could lead to false conclusions about certain products formed that are generated in the FAPA source and not in the EC cell. To verify this hypothesis, all samples have been additionally measured with standard EC-ESI-MS and the results of both methods were compared. Until now the method of choice for the detection of the products generated in electrochemical cell has been electrospray, and therefore such verification should be an important element of LC-FAPA development. Additional experiments did not indicate any "undefined" ion created in the gas phase of the FAPA source. All ions described in Table 1 were also seen in the ESI source (data not shown) what confirms that ions presented in the table are indeed products of the EC cell.

Due to the ongoing development of novel ion sources, there is continuous progress in mass spectrometry and its capabilities, for example progress from FAB to ESI and MALDI, and further to DESI, LAESI, etc. As DESI/LAESI employs similar ionization principles as ESI and MALDI, respectively, there seems to be little reason to explore them. In contrast, we consider FAPA to be complementary to ESI (or APCI or GC-MS), even though in many cases FAPA provides faster analysis, because it requires little or no sample preparation. Additionally, FAPA is advantageous because it can analyze solid samples directly and can serve as an imaging device (these features are currently being explored in our laboratory). The possibility of linking FAPA and a solution introduction system (here, CE) was the main goal of this study, which means that EC could be replaced by any other liquid-based separation system.

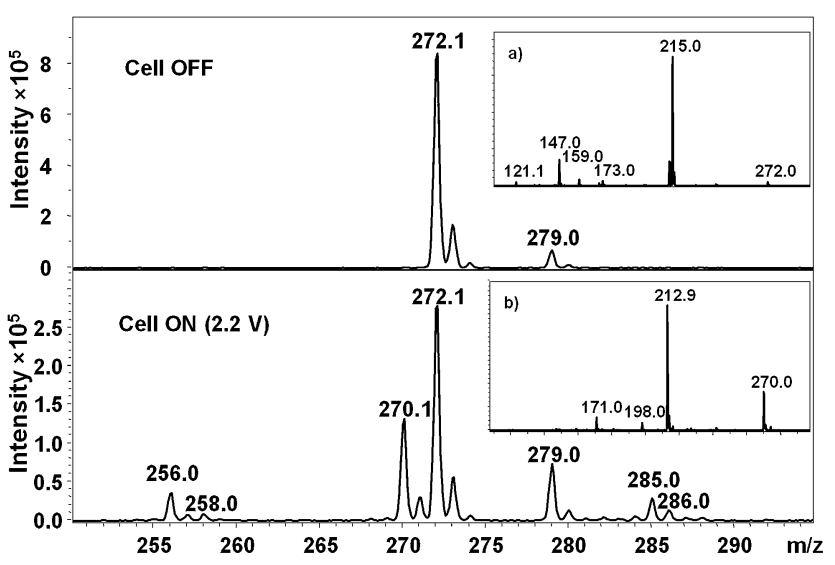

Fig. 4 FAPA mass spectrum of dextromethorphan $(\mathrm{m} / \mathrm{z}=272.1)$ before and after reaction in the electrochemical cell. For a detailed explanation see the text. The insets represent MS/MS spectra of (a) ion at $\mathrm{m} / \mathrm{z} 272.1$ corresponding to protonated dextromethorphan and (b) ion at $\mathrm{m} / \mathrm{z} 270.1$ corresponding to the dehydrogenated compound. The mass difference of $2 \mathrm{~m} / \mathrm{z}$ units for the main fragment ions is helpful in determining the site of dehydrogenation. The fragmentation pattern for DXM has been described elsewhere. ${ }^{35}$ 


\section{Conclusions}

The foregoing experiments reveal that an on-line combination of electrochemistry and flowing atmospheric pressure afterglow mass spectrometry can be a useful tool for quantitative analysis of psychostimulants and their potential metabolites, and is suitable as a step towards predictive toxicology, where rapid information on toxic metabolites of the novel legal highs and designer drugs may contribute to more effective medical treatment. The presented experiments confirm the possibility of simulating cellular metabolism of psychoactive compounds, based on dehydrogenation and N-deacylation, both of which are major pathways catalyzed by cytochrome P450 in humans and animals. The present work also illustrates the capabilities of the FAPA source to accept flowing liquid systems, which potentially opens up wider possibilities for applications not available to date.

\section{Acknowledgements}

The authors acknowledge support for this work in part by the U.S. Department of Energy through grant FG02-98ER14980, EuroNanoMed grant 5/EuroNanoMed/2012 grant, and by the Polish National Science Center the grant DEC-2012/07/N/ST4/ 01837.

\section{References}

1 F. J. Andrade, J. T. Shelley, W. C. Wetzel, M. R. Webb, G. Gamez, S. J. Ray and G. M. Hieftje, Anal. Chem., 2008, 80, 2654-2663.

2 F. J. Andrade, J. T. Shelley, W. C. Wetzel, M. R. Webb, G. Gamez, S. J. Ray and G. M. Hieftje, Anal. Chem., 2008, 80, 2646-2653.

3 J. T. Shelley, J. S. Wiley and G. M. Hieftje, Anal. Chem., 2011, 83, 5741-5748.

4 M. C. Jecklin, G. Gamez and R. Zenobi, Analyst, 2009, 134, 1629-1636.

5 R. B. Cody, J. A. Laramée and H. D. Durst, Anal. Chem., 2005, 77, 2297-2302.

6 N. Na, M. Zhao, S. Zhang, C. Yang and X. Zhang, J. Am. Soc. Mass Spectrom., 2007, 18, 1859-1862.

7 J. D. Harper, N. A. Charipar, C. C. Mulligan, X. Zhang, R. G. Cooks and Z. Ouyang, Anal. Chem., 2008, 80, 9097-9104.

8 M. C. Jecklin, S. Schmid, P. L. Urban, A. Amantonico and R. Zenobi, Electrophoresis, 2010, 31, 3597-3605.

9 J. T. Shelley and G. M. Hieftje, Analyst, 2010, 135, 682-687.

10 S. S. Johansen and H. M. Bhatia, J. Chromatogr. B: Anal. Technol. Biomed. Life Sci., 2007, 852, 338-344.

11 P. Pellinen, L. Kulmala, J. Konttila, S. Auriola, M. Pasanen and R. Juvonen, Arch. Toxicol., 2000, 74, 511-520.

12 H. Raoof, P. Mielczarek, K. A. Michalow, M. Rekas and J. Silberring, Biology, 2013, 118, 49-57.

13 H. Raoof, K. A. Michalow and J. Silberring, Drug metabolism simulation using $\mathrm{TiO}_{2} / \mathrm{UV}$ system, in
Biomacromolecular Mass Spectrometry: Tips from the Bench, ed. S. König, Nova Science Publ., 2012, pp. 139-152, ISBN 978-1-62081-932-6.

14 W. Lohmann, A. Baumann and U. Karst, $L C \cdot G C$ Eur., 2010, 1-6.

15 A. Baumann and U. Karst, Expert Opin. Drug Metab. Toxicol., 2010, 6, 715.

16 S. Jahn and U. Karst, J. Chromatogr. A, 2012, 1259, 16-49.

17 A. Baumann, W. Lohmann, B. Schubert, H. Oberacher and U. Karst, J. Chromatogr. A, 2009, 1216, 3192-3198.

18 P. Liu, M. Lu, Q. Zheng, Y. Zhang, H. D. Dewald and H. Chen, Analyst, 2013, 138, 5519-5539.

19 P. Suder, A. Bodzon-Kulakowska, P. Mak, A. BierczynskaKrzysik, M. Daszykowski, B. Walczak, G. Lubec, J. H. Kotlinska and J. Silberring, J. Proteome Res., 2009, 8, 4633-4640.

20 P. Suder, A. Bogusiewicz, K. Rolka, P. Laidler, J. Kotlinska and J. Silberring, Neurosci. Lett., 2003, 348, 167-170.

21 M. Chen, J. Zhang, Y. Wang, Z. Liu, R. Kelly, G. Zhou, H. Fang, J. Borlak and W. Tong, Clin. Pharmacol. Ther., 2013, 93, 409-412.

22 J. A. Marusich, T. W. Lefever, S. P. Novak, B. E. Blough and J. L. Wiley, Methods Rep. RTI Press, 2013, 1-14.

23 M. Smoluch, E. Reszke, A. Ramsza, K. Labuz and J. Silberring, Rapid Commun. Mass Spectrom., 2012, 26, 1577-1580.

24 M. Smoluch, E. Reszke, A. Ramsza and J. Silberring, in Biomacromolecular Mass Spectrometry: Tips from the Bench, ed. S. König, Nova Science Publ., 2012, ISBN 978-1-62081933-3.

25 S. M. van Leeuwen, B. Blankert, J. M. Kauffmann and U. Karst, Anal. Bioanal. Chem., 2005, 382, 742-750.

26 J. A. Birbeck and T. A. Mathews, Anal. Chem., 2013, 85, 73987404.

27 A. Kraft, Int. J. Electrochem. Sci., 2007, 2, 355-385.

28 J. T. Shelley, J. S. Wiley and G. M. Hieftje, Anal. Chem., 2011, 83, 5741-5748.

29 R. F. Staack, D. S. Theobald, L. D. Paul, D. Springer, T. Kraemer and H. H. Maurer, Drug Metab. Dispos., 2004, 32, 379-381.

30 R. F. Staack, J. Fehn and H. H. Maurer, J. Chromatogr. B: Anal. Technol. Biomed. Life Sci., 2003, 789, 27-41.

31 L. J. Schep, R. J. Slaughter and D. M. Beasley, Clin. Toxicol., 2010, 48, 675-694.

32 C. K. Yeung and A. E. Rettie, Methods Mol. Biol., 2006, 320, 157-162.

33 S. Kataoka, T. Naito, K. Nishimura and K. Taira, Chem. Pharm. Bull., 1979, 27, 2904-2912.

34 A. Yu and R. L. Haining, Drug Metab. Dispos., 2001, 29, 15141520.

35 T. H. Eichhold, D. L. McCauley-Myers, D. A. Khambe, G. A. Thompson and S. H. Hoke, J. Pharm. Biomed. Anal., 2007, 43, 586-600. 